\title{
Um Marcador Anatômico e a Proposta de Tratamento Cirúrgico na Síndrome do Cólon Irritável
}

\author{
Anatomical Marker and a Surgical Option in the Irritable Bowel \\ Syndrome Treatment
}

\author{
JÚLIO CÉSAR M. SANTOS JR ${ }^{1}$;ANACAROLINACAVALCA²; CARLOS ENRIQUE QUIROZ ${ }^{3}$ \\ ${ }^{1}$ Departmento de Cirurgia Geral - Secção de Cirurgia Colorretal; ${ }^{2}$ Departmento de Cirurgia Geral - Secção de \\ Ginecologia; ${ }^{3}$ Departmento de Radiologia - Centro de Diagnóstico do Hospital e Maternidade Frei Galvão - \\ Guaratinguetá - São Paulo - Brasil.
}

SANTOS JR JCM; CAVALCA AC; QUIROZ CE. Um Marcador Anatômico e a Proposta de Tratamento Rev bras Coloproct, 2006;26(3): 300-309.

RESUMO: Objetivo: A Síndrome do cólon irritável (SCI) é um distúrbio funcional caracterizado por dor abdominal e mudança de hábito intestinal. .Observando que a Síndrome do ceco móvel (SCM) engloba sintomas que se superpõem aos da SCI, nossos objetivos, foram: a. demonstrar a relação de causa e efeito entre o ceco móvel e a SCI, b. avaliar os resultados da cecopexia, como método de tratamento de pacientes com síndrome do cólon irritável (SCI) ou com desconforto abdominal de etiologia desconhecida; e c. mostrar que o ceco móvel pode ser considerado como o primeiro marcador anatômico para a SCI. Pacientes e Métodos: De março de 1994 a abril de 2006, 123 pacientes, receberam o diagnóstico clínico de ceco móvel. Destes, 103 (83,7\%) vinham sendo acompanhados e medicados como pacientes com SCI e 20 (16\%) vinham sendo tratados por outras doenças, Todos os pacientes desta série foram programados para cecopexia; contudo, 30 recusaram e estão sendo observados, 93 concordaram com o tratamento cirúrgico proposto. Sessenta e quatro foram operados e 29 aguardam cirurgia. Resultados: Todos os pacientes operados (64/52\%) foram acompanhados no pós-operatório, de 2 a 139 meses (média de 21 meses; dp= 23) e estão assintomáticos. Entre 58 mulheres operadas, 48 (82,7\%) responderam inquérito sobre dispareunia; entre essas, 44 (92\%) tinham dispareunia profunda. Após o tratamento cirúrgico, 89,7\% participaram do inquérito; $52(96 \%)$ estão assintomáticas. Os pacientes não operados $(59 / 48 \%)$ foram, da mesma forma, acompanhados de 2 até 72 meses e apresentavam os mesmos sintomas. Conclusão: Há nítida superposição de sintomas entre SCI e SCM; por isso recomendamos que: 1. a ênfase que se tem dado à SCI seja revista; 2. os pacientes com sintomas intestinais funcionais, quer sejam os atribuíveis à SCI, quer sejam os de causas não esclarecidas, e os pacientes com dispareunia de etiologia obscura devem ser investigados como prováveis portadores da SCM; 3. O ceco móvel pode ser usado como indicador anatômico da SCI.

Descritores: Síndrome do cólon irritável, síndrome do ceco móvel, plenitude abdominal, cólica abdominal, constipação, diarréia, torção do ceco, dispareunia, cecopexia.

A síndrome do cólon irritável (SCI) é um distúrbio funcional caracterizado por dor abdominal e mudanças no hábito intestinal, não associadas com qualquer anomalia vista em provas clínicas rotineiras. ${ }^{1,2}$

Há duas formas principais da SCI, na dependência do sintoma predominante: SCI com dor abdo- minal baixa e dispepsia não-ulcerosa em que os pacientes podem ter constipação (SCIC) ou diarréia (SICID), ou pode apresentar constipação e diarréia flutuantes (SICICD). Há, no entanto, um novo subtipo sob investigação denominado de síndrome do cólon pósinfecciosa (SCIPI).

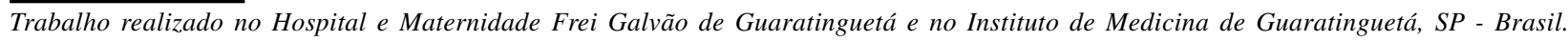


Um Marcador Anatômico e a Proposta de Tratamento

Cirúrgico na Síndrome do Cólon Irritável

Júlio César M Santos Jr. e Cols.
Vol. 26
Trata-se de entidade de alta prevalência e responsável por $20-50 \%$ de visitas ao gastrenterologista. Apesar de ser comum, com incidência populacional de até 30\% (predominando nas mulheres), e de ser alvo de estudos com destaque para diversos hormônios de ação gastrintestinal ${ }^{3-11}$, o diagnóstico e o tratamento dessa doença nem sempre são tarefas fáceis de serem executadas, sobretudo pela falta de um marcador que defina sua natureza, não só para retirá-la da classe de "doença de diagnóstico de exceção", como para permitir opções terapêuticas efetivas. ${ }^{12,13}$

Baseados na observação de que a Síndrome do ceco móvel (SCM) engloba sintomas que se superpõem aos da SCI ${ }^{14}$ nossos objetivos, ao desenvolver um estudo prospectivo não casualizado, foram: a. demonstrar a relação de causa e efeito entre o ceco móvel e a SCI, b. avaliar o resultado da cecopexia, como método de tratamento de pacientes com Síndrome do cólon irritável (SCI) ou com desconforto abdominal de etiologia desconhecida; e c. mostrar que o ceco móvel pode ser considerado como o primeiro marcador anatômico para a SCI.

\section{PACIENTES E MÉTODOS}

Foram incluídos neste estudo: 1. todos os pacientes vistos em duas Clínicas Privadas (de Ginecologia e Obstetrícia e de Colo-Proctologia) que preencheram os critérios para diagnóstico de SCI (Manning, Roma I e II) ${ }^{15-16} 2$. todos os pacientes com o diagnóstico clínico de SCI ${ }^{16,17}$ oriundos de outros consultórios médicos; e 3. os pacientes sem um diagnóstico formal, mas que se queixavam de dor abdominal de etiologia desconhecida, cólica, constipação, diarréia, ou diarréia e/ou constipação flutuantes; distensão ou plenitude abdominal e sensação de evacuação incompleta, que tinham ou não dor abdominal durante as relações sexuais.

Assim, de março de 1994 a abril de 2006, 123 pacientes (109 mulheres e 14 homens) com idade média de 37,7 anos (variando de 3 a 82 anos), a maioria deles $103(83,7 \%)$ registrados como tendo SCI e 20 (16,3\%) com suspeita clínica de doenças como: dor abdominal inespecífica, doenças ginecológicas, doença intestinal inflamatória, colecistopatia calculosa, doença diverticular, apendicite, megacólon e giardíase, foram investigados e, clinicamente, considerados com o ceco móvel.

O diagnóstico de ceco móvel foi confirmado radiologicamente em 107 pacientes (87\%); em 98 pa- cientes $(79,7 \%)$ por meio de técnica modificada de trânsito intestinal e em 8 pacientes $(6,5 \%)$, pelo enema opaco. Nos 17 pacientes $(13,8 \%)$ restantes, o diagnóstico foi exclusivamente clínico, confirmado no intraoperatório em 10 deles, que foram operados.

$\mathrm{O}$ trânsito intestinal foi feito com a seguinte técnica: os pacientes foram orientados a ingerir $40 \mathrm{ml}$ de uma refeição de bário (as crianças, $20 \mathrm{ml}$ ), às 8 horas da manhã, sem preparo prévio ou alteração da alimentação habitual, seja na véspera ou no dia do exame.

Radiografias abdominais, incluindo a pequena bacia, foram tomadas depois de 5 e 10 horas da refeição de bário, nas posições ortostática e em decúbito dorsal com declive cefálico e pés elevados, numa inclinação de 15 graus.

As imagens radiográficas foram analisadas sempre pelo mesmo radiologista e classificadas, quanto à mobilidade do ceco em relação à pelve, em tipo I, II, III e IV, de acordo como o que foi proposto por Padrón e Ania ${ }^{18}$, depois modificado por Santos e col. ${ }^{19,20}$, como segue:

Tipo I - Quando o paciente está em decúbito dorsal, o ceco é visto na situação anatômica normal, mas cai em direção à pequena bacia quando o paciente está em pé.

Tipo II - No decúbito dorsal, o ceco se encontra abaixo de sua situação anatômica normal e tem uma descida evidente quando o paciente fica em pé.

Tipo III - Com o paciente em pé, o ceco é visto dentro da pelve, de onde não se move quando o paciente é posto em posição de decúbito dorsal.

Tipo IV - No decúbito dorsal, o ceco está numa situação anatômica normal ou acima da posição e o ângulo hepático do cólon é visto no rebordo costal ou um pouco abaixo. Quando o paciente fica em pé, o ceco, o cólon ascendente, o ângulo hepático e o cólon transverso ficam todos dentro da bacia.

As figuras de 1, 2 (báscula medial do ceco) e 5 (báscula lateral do ceco) são exemplos de enemas opacos; as figuras 3 e 4 são imagens tipo IV obtidas pelo transistor intestinal.

Independentemente da gradação radiológica do ceco móvel, os pacientes foram aconselhados para o tratamento que consistiria da cecopexia e, de acordo com a aceitação ou não da proposta, eles foram separados em dois subgrupos denominados de pacientes não operados (PNO) e pacientes que aceitaram a operação (PAO). Nesse último subgrupo há os pacientes 


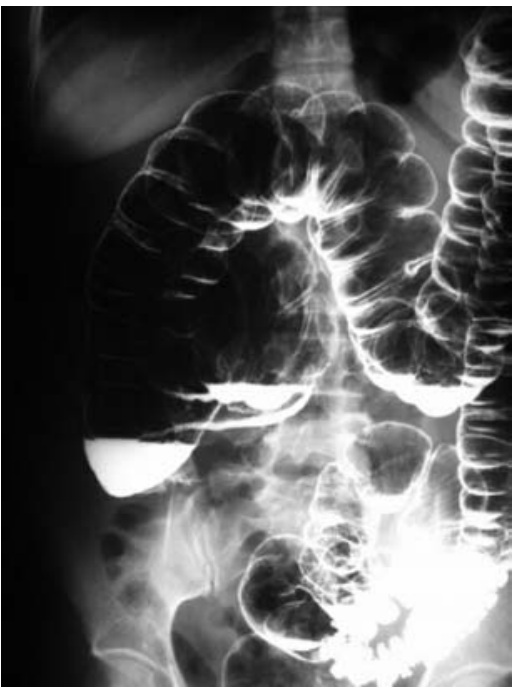

Figural - Enema opaco.

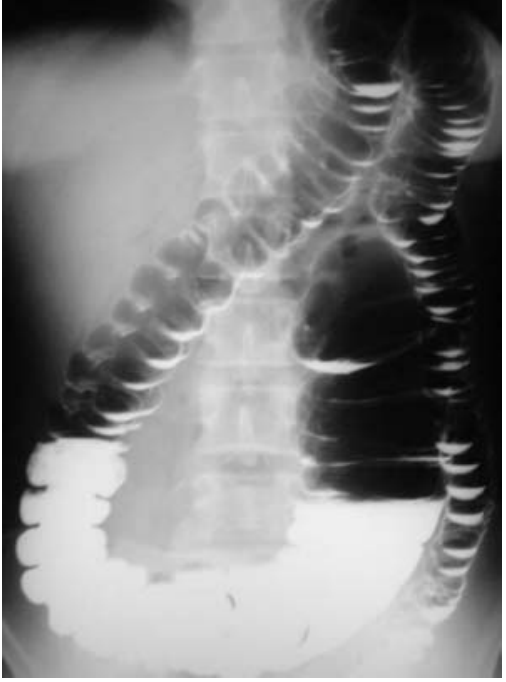

Figura 2 - Enema opaco.
Vol. 26 No 3

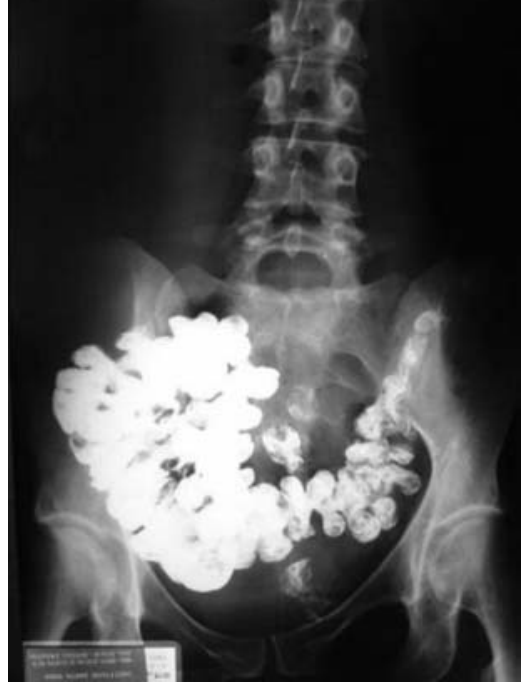

Figura 3 - Trânsito - ortostático.

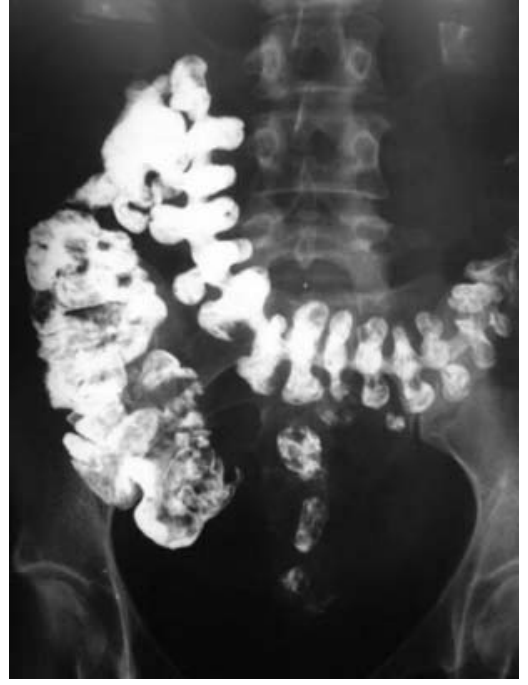

Figura 4 - Trânsito decúbito dorsal.

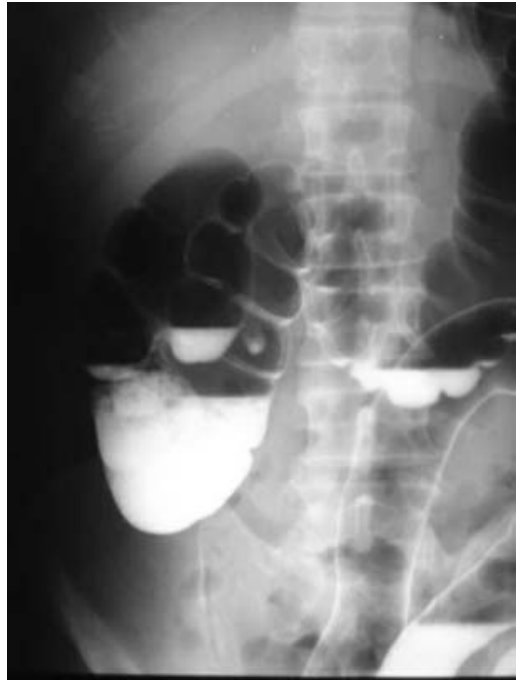

Figura 5 - Enema opaco - báscula lateral do ceco. que já foram operados (PO) e os que aguardam data oportuna para $\mathrm{o}$ ato cirúrgico (PAC).

Os pacientes que recusaram o tratamento cirúrgico, aceitaram, no entanto, o tratamento clínico. Esses pacientes, após o diagnóstico definitivo do ceco móvel, suposto como causa fundamental dos sintomas, foram mantidos com o tratamento anteriormente iniciado para síndrome do cólon irritável, considerando-se a presença ou não de constipação ou de diarréia, ou da associação de constipação com diarréia, e a intensidade das manifestações, de acordo com o que tem sido discutido e preconizado. ${ }^{2,12,21-23}$
Eles foram orientados para serem vistos quatro vezes por ano, nos dois primeiros anos, após o contato inicial conosco, e duas vezes por ano, a partir do terceiro ano, com direito a retornos livres, em qualquer época, se necessário.

Os pacientes que aceitaram a operação (PAO) e foram operados $(\mathrm{PO})$ receberam orientação para retorno no sétimo dia do pós-operatório, no décimo quinto dia, no trigésimo dia, no sexto mês e no final de um ano; a partir de um ano, a cada quatro meses, ao longo do segundo e terceiro anos da operação e depois, duas vezes por ano. Os que aceitaram o trata- 
Um Marcador Anatômico e a Proposta de Tratamento

Cirúrgico na Síndrome do Cólon Irritável

Júlio César M Santos Jr. e Cols.
Vol. 26 $\mathbf{N}^{\mathbf{0}} 3$ mento cirúrgico, mas estão aguardando o momento oportuno para a cirurgia (PAC) estão recebendo o mesmo tratamento dado aos pacientes que recusaram o tratamento cirúrgico (PNO).

A operação cirúrgica foi feita com o paciente sob anestesia geral, por meio de minilaparotomias; nas crianças, a incisão foi a de Davis e nos adultos a incisão foi transversa suprapúbica, tipo Pfannenstiel. A ceco-colopexia foi feita como descrito por Rogers $^{24}$. Os pacientes adultos receberam alta hospitalar 24 horas depois da operação; as crianças, 48 horas.

Tanto no pré-operatório como no pós-operatório e nos seguimentos ambulatoriais regulares, todos os pacientes foram independentemente entrevistados, de forma padronizada, por dois de nós (Santos e Cavalca) a respeito de seu estado de saúde referente à doença que motivou a operação ou o estado atual de saúde referente à doença em tratamento clínico. Os dados obtidos da entrevista e da avaliação clínica ambulatorial foram registrados no prontuário médico de cada paciente.

No final de abril de 2006, 12 anos após a operação do primeiro paciente desta série, todos os pacientes receberam um questionário padrão (Anexo 1) para avaliação do estado de saúde na ocasião do inquérito, com perguntas sobre a persistência ou não dos sintomas que motivaram o tratamento cirúrgico ou o tratamento clínico, devolvido anônimo pelo correio.

O conjunto de respostas foi comparado com o conjunto dos dados obtidos e anotados nos prontuários médicos, por ocasião de entrevistas médicas nos retornos ambulatoriais.

As mulheres sexualmente ativas foram questionadas sobre dor durante o ato sexual, antes e depois do tratamento.

\section{RESULTADOS}

Anteriormente ao diagnóstico de síndrome do ceco móvel, 103 pacientes $(83,7 \%)$ vinham sendo tratados como doentes com Síndrome do cólon irritável e $20(16,3 \%)$ por outras doenças, tais como: doença inflamatória pélvica $(3,3 \%)$, dor abdominal inespecífica $(3,2 \%)$, doença diverticular do cólon $(2,4 \%)$, doença intestinal inflamatória $(2,4 \%)$, colecistopatia calculosa $(1,6 \%)$, apendicite, megacólon e giardíase $(3,2 \%)$.

Os 123 pacientes desta série relataram 427 sintomas, numa média aproximada de 3,5 sintomas por paciente, dentre os mais freqüentes foram: distensão, constipação, cólica, dor e diarréia - classificados, em primeiro, segundo, terceiro e quarto lugar, de acordo com a relevância do sintoma, ajuizada pelo paciente. Pelo menos três dos cinco sintomas mencionados foram referidos por todos os doentes. Cinquienta e oito pacientes $(47 \%)$ relataram quatro dos cinco sintomas mais frequientes (Tabela 1).

Cento e dezessete pacientes $(95 \%)$ reclamaram de distensão, 102 (83\%) de constipação, 90 (73\%) de cólica, $74(60 \%)$ de dor e $44(35 \%)$ de diarréia (Tabelas 1 e 2$)$.

Oitenta e oito por cento dos pacientes (109/ 123) relataram alívio dos sintomas imediatamente após a defecação, porém sem seu desaparecimento completo.

\section{Seguimento após definição do método de tratamento}

Cinqüenta e nove pacientes (48\%) - 30 (24\%) que recusaram o tratamento cirúrgico e 29 (23,6\%) que aguardam pela operação - foram orientados para serem vistos quatro vezes por ano, nos dois primeiros anos, após o contato inicial conosco e duas vezes por ano, a partir do terceiro ano, com direito a retornos

Tabela 1 - Sintomas graduados pelos pacientes por ordem de relevância.

\begin{tabular}{lccccrr}
\hline Sintomas & primeiro $\mathbf{n}(\%)$ & segundo $\mathbf{n}(\%)$ & terceiro $\mathbf{n}(\%)$ & quarton(\%) & Total $\mathbf{n}(\%)$ \\
\hline Distensão & $43(35)$ & $60(48,8)$ & $13(10,5)$ & - & $117(95)$ \\
Dor & $40(32)$ & $11(8,9)$ & $12(10)$ & 12 & $(9,8)$ & $75(61)$ \\
Constipação & $18(14,6)$ & $20(16,2)$ & $42(34)$ & $22(17,9)$ & $102(83)$ \\
Cólica & $17(13,8)$ & $28(22,8)$ & $31(25,2)$ & $14(11,4)$ & $90(73)$ \\
Diarréia & $5(4,1)$ & $4(3,3)$ & $25(20,3)$ & $10(8,1)$ & $44(35)$ \\
Total & 123 & 123 & 123 & $58(47)$ & 427 \\
\hline
\end{tabular}


Rev bras Coloproct Julho/Setembro, 2006
Um Marcador Anatômico e a Proposta de Tratamento

Cirúrgico na Síndrome do Cólon Irritável

Júlio César M Santos Jr. e Cols.
Vol. 26 $\mathbf{N}^{\mathbf{0}} 3$

Tabela 2 - Dados demográficos, sintomas mais freqüentes mencionados pelos pacientes; dispareunia mencionada pelas mulheres e os diagnósticos prévios anotados para o grupo todo e para o subgrupo de pacientes operados.

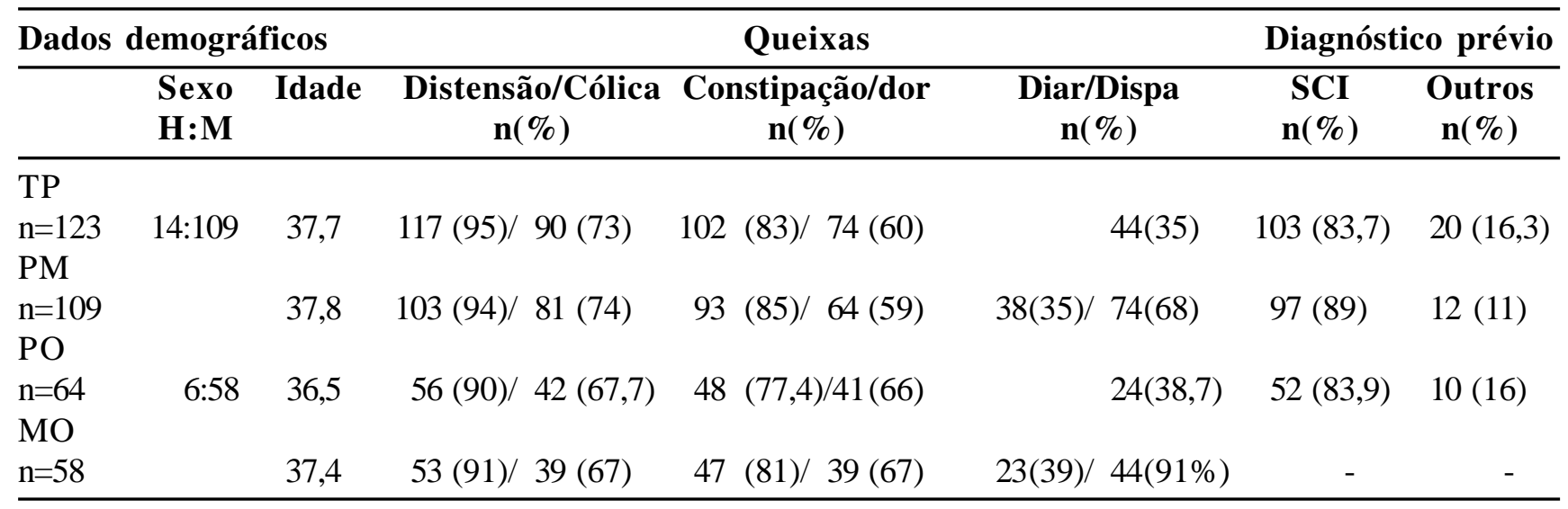

$T P=$ Todos pacientes; $P M=$ pacientes mulheres; $P O=$ operados; $M O=$ mulheres operadas; $S C I=$ síndrome do cólon irritável; $H=$ homem; $M=$ mulher $;$ Diar= diarréia $;$ Dispa $=$ dispareunia .

livres, em qualquer época, se necessário. Eles têm sido acompanhados desde 2 até 72 meses (média de 16 meses, $\mathrm{dp}=18$ ) - apresentando os mesmos sintomas, motivo da primeira consulta. Esses pacientes responderam o questionário (Anexo 1): 17(29\%), classificaram o estado atual de saúde como regular e os outros $42(71 \%)$ assinalaram como saúde inalterada em relação aos problemas que motivaram a primeira consulta. (Tabela 3). O conjunto de respostas dadas nos questionários não diferiu dos dados de entrevistas obtidos dos prontuários dos pacientes.

Dentre os 93 pacientes que concordaram com o tratamento cirúrgico proposto, 64 (69\%) já foram operados.

Todos os 64 pacientes operados estão sendo acompanhados no pós-operatório, de 2 a 139 meses (média de 21,6 meses; $\mathrm{dp}=24)$. Todos responderam o questionário (Anexo 1).

No grupo de pacientes operados, cinco pacientes $(8 \%)$ relataram a persistência de constipação, dois $(3 \%)$ classificaram o estado atual de saúde como regular e 62(97\%) classificaram o estado atual de saúde como bom, por estarem livres dos sintomas referidos antes da operação. (Tabelas 2 e 3 )

Cento e oito mulheres dessa série são sexualmente ativas. Questionadas, antes da operação, sobre dor abdominal durante o ato sexual, obtivemos o seguinte: $23(21 \%)$ não responderam o questionário; $85(79 \%)$ responderam. Entre estas $74(87 \%)$ responderam sim e 11(13\%) disseram não, significando que pelo menos $68 \%$ das mulheres tinham dispareunia. (Tabela 2)

Tabela 3 - Dados demográficos, tempo de seguimento e resultados obtidos com o questionário (Anexo 1), exceto a dispareunia.

\begin{tabular}{|c|c|c|c|c|c|c|c|c|}
\hline \multicolumn{3}{|c|}{ Dados demográficos } & \multicolumn{4}{|c|}{ Atual estado de saúde em relação ao tratamento } & \multicolumn{2}{|c|}{ Seguimento (meses) } \\
\hline & $\begin{array}{l}\text { Sexo } \\
\text { H:M }\end{array}$ & $\begin{array}{l}\text { Idade } \\
\text { média }\end{array}$ & $\begin{array}{l}\text { Bom } \\
\text { n(\%) }\end{array}$ & $\begin{array}{c}\text { Regular } \\
\text { (n\%) }\end{array}$ & $\begin{array}{c}\text { Inalterado } \\
\mathbf{n}(\%)\end{array}$ & Pior & Global & PO \\
\hline $\begin{array}{l}\text { OP } \\
n=64 \\
N P \\
n=59\end{array}$ & $6: 58$ & 36,4 & $62(97)$ & $2(3)$ & $42(71)$ & 0 & $\begin{array}{cc}21,6 & (3-143) \\
& d p=24 \\
16 & (1-72) \\
& d p=18\end{array}$ & $\begin{array}{c}19,(30-139) \\
\mathrm{dp}=23\end{array}$ \\
\hline
\end{tabular}

$O P=$ operado $; N P=$ não operado $; H=$ homem $; M=$ mulher $; P O=$ pós-operatório $; d p=$ desvio padrão. 
Das cinqüienta e oito mulheres operadas, quando questionadas no pré-operatório sobre dispareunia, 10 $(17,2 \%)$ não responderam. Quarenta e oito mulheres responderam: $44(91,7 \%)$ haviam dito sim e $4(8 \%)$ haviam dito não.

Durante o seguimento pós-operatório elas foram, novamente, questionadas a respeito da dispareunia; 52 delas $(89,6 \%)$ responderam; 50 (96\%) disseram não e $2(4 \%)$ disseram sim.

\section{DISCUSSÃO}

A SCI é doença altamente prevalente, mas a despeito de todo conhecimento e teorias formuladas a respeito da sua fisiopatologia, ainda não temos um agente etiológico claro e bem estabelecido para caracterizá$\mathrm{la}^{1}$. Fatores culturais e decorrentes de hábitos alimentares têm sido isolados com sugestões de agentes etiológicos. ${ }^{25,26}$

Hiperalgesia visceral, estresse, abuso físico e sexual têm sido elementos associados como participantes do conjunto de dados clínicos, englobado no universo dessa moléstia ${ }^{1-9}$, da mesma forma ocorrendo com as enterites bacterianas e virais, sem que, no entanto, seja possível destacar um elemento marcador. ${ }^{27,28}$

São tantas as teorias e complexas as investigações e conclusões que a SCI passa a ser vista sob um conjunto heterogêneo de desordens com sintomas parecidos, mas de diferentes etiologias ${ }^{29-32}$ que retroalimentam mais especulações sobre uma causa básica, o que, sem dúvida alguma, favorece a "medicalização" e a emergência de procedimentos subsidiados pela medicina alternativa ${ }^{33}$.

Em 1979, demos assistência ao ato operatório de uma paciente com SCI que, além de distúrbios intestinais funcionais, se queixava de dor pélvica, na ocasião atribuída a aderências intestinais relacionadas à histerectomia, feita no passado. Durante a exploração metódica da cavidade, o único achado, pelo qual me chamou o cirurgião ginecólogo que a operava, foi a livre movimentação do segmento ceco-cólon ascendente da paciente. $\mathrm{O}$ ato cirúrgico terminou com a fixação ceco-ascendente numa calha feita após a abertura do peritônio na goteira parietocólica direita. A surpresa em relação a essa ocorrência foi a resolução dos problemas intestinais e o desaparecimento das dores pélvicas da paciente. $\mathrm{O}$ fato foi inusitado, mas por atribuir toda a sintomatologia da paciente a outros fatores, inclusive aos de ordem psicológica, não fomos capazes de con- siderar a relevância do efeito de ação e reação.

Em 1994, no início do exercício médico como profissional liberal, ao atender a nossa primeira cliente, deparamo-nos com uma história clínica de 5 anos típica da SCI, tipo constipação/diarréia flutuantes, doença para a qual a paciente vinha recebendo tratamento médico ineficaz. Iniciamos a investigação para excluir outras doenças e o primeiro exame solicitado foi o enema opaco pelo qual se pôde diagnosticar a báscula medial do ceco (Figura 6). Seguiu-se, ao tratamento cirúrgico, o desaparecimento dos sintomas crônicos de disfunção intestinal.

A partir de então, passamos a fazer investigação topográfica do segmento ceco-ascendente em todos os pacientes com diagnóstico de SCI e em pacientes com desconforto abdominal de causa obscura, independente do sexo e da idade.

O constante foi a verificação de que todos tinham ceco móvel de grau variado. Seria este um fator coincidente, ou o ceco móvel - doença antiga, comum e ordinariamente mal diagnosticada ${ }^{34,35}$, já descrito como uma síndrome ${ }^{24}$ - poderia ser usado para explicar os mesmos elementos sintomatológicos da SCI?

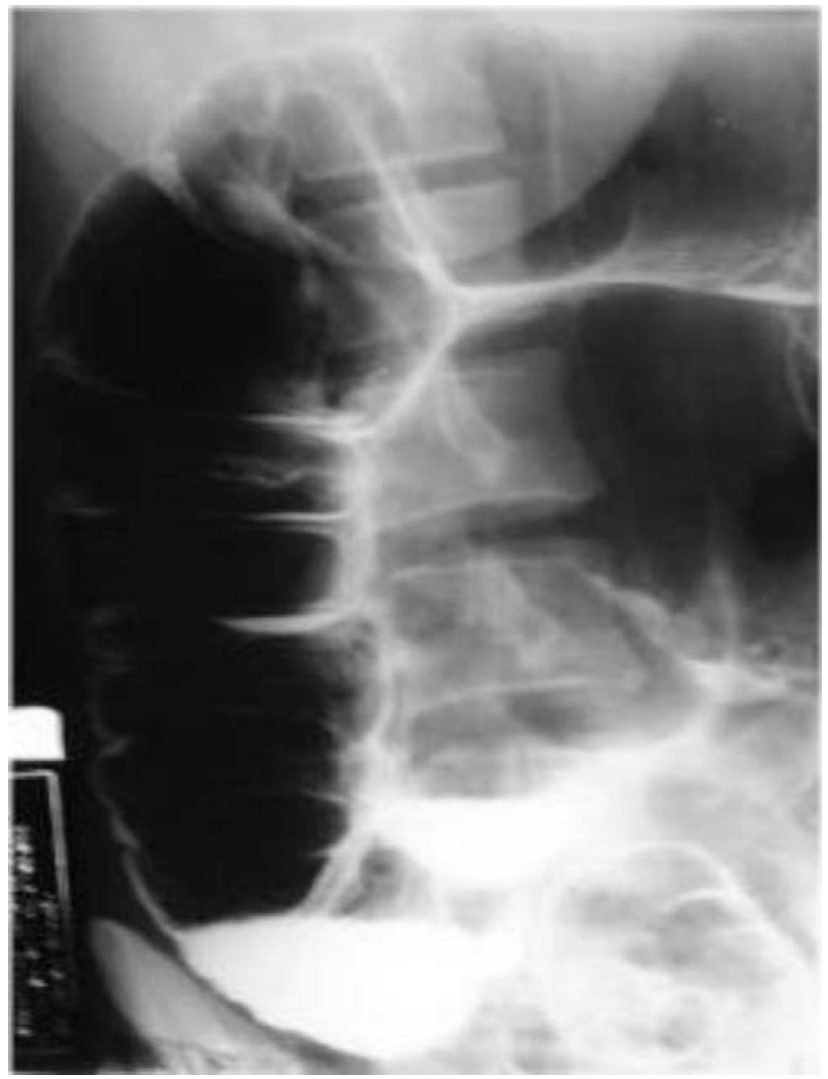

Figura 6 - Enema opaco - 1a paciente dessa série. 
Um Marcador Anatômico e a Proposta de Tratamento

Cirúrgico na Síndrome do Cólon Irritável

Júlio César M Santos Jr. e Cols.
Vol. 26
Considerando os dados observados nesse estudo e inclinados à reflexão, iniciamos sugerindo que a maioria dos pacientes diagnosticados como tendo SCI na verdade não sofrem de SCI, mas, sim, de SCM. Contudo, se deixarmos de lado a complexidade das investigações sobre a SCI e as eloqüentes e especulativas teorias a respeito da sua fisiopatologia e etiopatogenia, ainda que necessitemos de comprovação de outras fontes feitas por outros autores, somos inclinados a dizer que, quando mencionamos SCI e SCM, estamos falando da mesma doença e a variação anatômica congênita do ceco e cólon ascendente (CID-10 Q43.3) seria a base na qual uma doença alicerça a outra.

Há uma observação interessante, adicionada nessa comunicação; depois de ter elaborado todo nosso raciocínio a respeito de ceco móvel e distúrbios intestinais funcionais, que foi motivada por Tirol ${ }^{36}$ quando descreveu relação entre ceco móvel e dor abdominal durante as relações sexuais, o que ele denominou de "dispareunia do lado direito", sintoma erradicado com a cecopexia.

O autor deu ênfase ao fato do ceco móvel poder ser a causa da dor crônica ou intermitente e pelo desconforto abdominal periódico, localizado no quadrante inferior direito do abdômen, sentidos durante a relação sexual. Além disso, fez comentários sobre a alta e significante incidência do problema, seus sintomas e sinais, o fácil diagnóstico e tratamento cirúrgico, bem como apelou para o maior interesse do médico no reconhecimento da Síndrome do ceco móvel. ${ }^{37,38}$

A incidência de dispareunia entre as mulheres sexualmente ativas em nosso grupo, definida por outros como psicogênica, foi considerada alta. Setenta e oito por cento das mulheres participaram da primeira enquête; $87 \%$ delas relataram dispareunia. $\mathrm{O}$ valor percentual de participação aumentou para $83 \%$, na segunda enquête e, nessa ocasião, 92\% das mulheres referiam a dor abdominal no intercurso sexual.

Após a operação, durante o seguimento ambulatorial, 89,7\% participaram do questionário; 50 (96\%) disseram não e 2 (4\%) disseram sim, evidência da eficácia do tratamento cirúrgico que precisa ser confirmada.

Esses dados são sugestivos de falta de adesão dos pacientes quando o tema investigado origina em questões que interferem com a sensibilidade das pessoas, mas mostra, também, a alta incidência de distúrbio da esfera sexual, supostamente sem causa orgânica, associado às alterações topográficas do ceco. Fo- ram 58 mulheres operadas, todas sexualmente ativas seis não quiseram responder sobre questão da intimidade sexual - mas das 52 que sentiam dor abdominal durante a relação sexual, 50 (96\%) ficaram livres do problema após a cecopexia.

Todos os pacientes do presente estudo apresentaram, também, os sintomas de perturbações gastrintestinais funcionais, com destaque para as reclamações de dor abdominal intermitente, não necessariamente localizada no quadrante inferior direito do abdômen; cólica, constipação, diarréia ou constipação seguida de diarréia; distensão abdominal ou inchaço.

Anteriormente ao estudo, os pacientes foram vistos e examinados por vários médicos, entre gastrenterologistas e ginecologistas, e submetidos a uma multiplicidade de exames, tais como ultra-som abdominal, tomografia abdominal, enema opaco, colonoscopia, exames ginecológicos, entre outros, quase sempre sem que qualquer anomalia fosse encontrada.

Devido aos sintomas mencionados, quase todos os pacientes $(103 / 83,7 \%)$ foram vistos e tratados como tendo Síndrome do cólon irritável e, porisso, lhes foram administrados um ou mais de um dos seguintes medicamentos: brometo de pinaverium, mebeverine, cisaprida, tegaserode, plantago ovata, brometo de Nbutilscopolamine, associados ou não ao clonazepam para suavizar os sintomas intermitentes e crônicos.

Quando o diagnóstico de Síndrome de ceco móvel substituiu o de Síndrome do cólon irritável, o cuidado médico foi consequientemente alterado e a terapêutica clínica foi substituída pela cirúrgica, o que possibilitou a erradicação do problema, inclusive a dispareunia, como mencionado.

Na maioria dos mais recentes trabalhos encontrados na literatura médica em que há menção ao ceco móvel, se não em todos, excluindo os de Tirol ${ }^{34-38}$, os autores apenas mencionam os aspectos associados com a oclusão intestinal parcial, que pode ser devida à báscula cecal, à torção parcial e intermitente daquele segmento do intestino grosso ou à torção completa que pode culminar com obstrução do cólon direito e necrose do ceco. Nesses casos, o tratamento cirúrgico urgente proposto, em geral, termina com a excisão de parte do cólon direito ${ }^{24,39-49}$.

Não há descrita nenhuma relação entre ceco móvel e desordens intestinais funcionais; especialmente, não houve ainda menção à Síndrome de cólon irritável com o atual enfoque, embora Tirol ${ }^{36}$ tenha descrito uma relação entre dispareunia e ceco móvel. 
Rev bras Coloproct Julho/Setembro, 2006
Um Marcador Anatômico e a Proposta de Tratamento Cirúrgico na Síndrome do Cólon Irritável Júlio César M Santos Jr. e Cols.
Vol. 26 $\mathbf{N}^{\circ} 3$
Nosso primordial objetivo com essas observações foi o de estabelecer relação entre a anomalia congênita - conhecida, mas pouco reconhecida e mal diagnosticada - e a Síndrome do cólon irritável, ressaltando, por isso, o fato de que a ênfase que tem sido dada à SCI precisa ser revista. Além disso, como já foi observado por Tirol ${ }^{36}$, a dispareunia profunda, excluídas todas as outras causas e antes de se lhe atribuir etiológica psicológica, deve ser relacionada ao ceco móvel e, portanto, considerada como um sintoma a mais no conjunto dos elementos clínicos que favorecem o diagnóstico daquela síndrome.

Se nossas observações puderem ser corroboradas por outros autores, certamente estaremos diante de um marcador anatômico para a SCI e a maioria dos pacientes, se não todos, com essa síndrome serão beneficiados pela cecopexia.

Em suma, baseados nos resultados, concluímos que: 1. a investigação topográfica do ceco-ascendente deve ser feita para todos os pacientes com diagnóstico de SCI ou com sintomas abdominais funcionais obscuros; 2. os pacientes, dessa série, com SCI tinham ceco móvel e foram curados com cecocolopexia; 3 . há relação de causa e efeito entre ceco móvel e SCI; 4. o ceco móvel pode ser apontado como o primeiro indicador anatômico para a SCI, e 5. o ceco móvel pode ser considerado como causa de dispareunia profunda.

\begin{abstract}
Purpose: Based on the observation that the mobile cecum syndrome (MCS) includes symptoms which overlap symptoms of the IBS, our purposes were: to demonstrate the relationship of cause and effect between the mobile cecum and IBS; to evaluate the result of the cecopexia, as method of patients' treatment with irritable bowel syndrome (IBS) or with abdominal discomfort of ignored etiology; and to show that the mobile cecum can be considered as the first anatomical marker for IBS. Patients and Methods: From 1994 to 2006, 123 patients (109 women and 14 men - median age, 37,7 - ranged 3 to 82 ) seen in private office had clinical diagnosis of mobile cecum; 103 patients $(83,7 \%)$ had been seen as having irritable bowel syndrome. All patients of this series were programmed for cecopexy. Thirty refused the surgical treatment and 29 are waiting for suitable surgery; both are on clinical follow-up in private office Results: All operated patients (64/52\%) with fixed cecum followed from 1 to 139 months (median, 21) are well and asymptomatic Among the 58 operated women, $48(82,7 \%)$ answered the inquiry about dispareunia; 44(92\%) had dispareunia. After surgical treatment $89,7 \%$ have participated of the inquiry; $52(96 \%)$ were without symptoms. The non-operated patients $(59 / 48 \%)$ followed from 1 to 72 months (median, 16) are with the same complain. Conclusion: Based on the results, we conclude that: the topographical investigation of the cecum-ascendent should be made for all the patients with diagnosis of IBS or with functional obscure abdominal symptoms; the patients of that serie with IBS had mobile cecum and they were cured with cecopexy; there is a relationship of cause and effect between mobile cecum and IBS; the mobile cecum can be pointed as the first anatomical marker for SCI; the mobile cecum can be considered as cause of deep dispareunia.
\end{abstract}

Key words: Mobile cecum syndrome, irritable bowel syndrome, intermittent abdominal complains, abdominal pain, colic, diarrhea, constipation, cecal volvulus.

\section{ANEXO 1}

$\operatorname{Sr}(\mathrm{a})$.

1. Antes de sua operação, dos cinco sintomas abaixo especificados, o(a) $\mathrm{Sr}$ (a) mencionou pelo menos três. Nós gostaríamos de saber se o $\operatorname{Sr}(a)$ poderia marcar quais deles ainda persistem.

$\begin{array}{ll}\text { o } & \text { Dor } \\ \text { o } & \text { Constipação } \\ \text { o } & \text { Cólica } \\ \text { o } & \text { Empachamento ou distensão } \\ \text { o } & \text { Diarréia } \\ \text { o } & \text { Nenhum }\end{array}$

2. Sendo mulher, responda-nos se antes da operação a Sra. tinha ou não dor ou desconforto abdominal durante as relações sexuais.

$$
\begin{array}{ll}
\text { o } & \text { Sim } \\
\text { o } & \text { Não }
\end{array}
$$

3. Sendo mulher, responda-nos se após a operação a Sra. continua tendo dor ou desconforto abdominal, durante as relações sexuais.

$\begin{array}{ll}\text { o } & \text { Sim } \\ \text { o } & \text { Não }\end{array}$


Rev bras Coloproct Julho/Setembro, 2006
Um Marcador Anatômico e a Proposta de Tratamento Cirúrgico na Síndrome do Cólon Irritável Júlio César M Santos Jr. e Cols.
Vol. 26 $\mathbf{N}^{\mathbf{0}} 3$
4. Tendo em vista os problemas que motivaram sua operação, nós poderíamos saber em que classe abaixo especificada está o seu atual estado de saúde?

$\begin{array}{ll}\text { o } & \text { Bom } \\ \text { o } & \text { Regular } \\ \text { o } & \text { Inalterado } \\ \text { o } & \text { Pior }\end{array}$

\section{REFERÊNCIAS}

1. Cash BD, Chey WD. Diagnosis of irritable bowel syndrome. Gastrenterol Clin North Am 2005;34:205-20.

2. Drossman DA, Thompson WG. The irritable bowel syndrome: review and a graduated multicomponent treatment approach. Ann Intern Med 1992;116: 1009-16.

3. Mayer EA. The neurobiology of stress and gastrointestinal disease. Gut 2000;47: 861-9.

4. Mawdsley JE, Rampton DS. Psychological stress in IBD: new insights into pathogenic and therapeutic implications. Gut 2005; 54:1481-91.

5. Kilkens TOC, Honig A, Van Nieuwenhoven MA. Acute tryptophan depletion affects brain-gut responses in irritable bowel syndrome patients and controls. Gut 2004; 53:1794-800.

6. De Ponti F. Pharmacology of serotonin: what a clinician should know. Gut 2004; 53:1520-35.

7. Thompson WG, Longstreth GF, Drossman DA. Functional bowel disorders and functional abdominal pain. Gut 1999; 45: suppl 2: II43-7.

8. Brandt LJ, Bjorkman D, Fennerty MB. Systematic review on the management of irritable bowel syndrome in North America. Am J Gastroenterol 2002; 97:S7-26.

9. De Ponti F, Tonini M. Irritable bowel syndrome: new agents targeting serotonin receptor subtypes. Drugs 2001; 61:317-32.

10. Crowell MD. The role of serotonin in the pathophysiology of irritable bowel syndrome. Am J Manag Care 2001; 7(suppl 8):S252-60.

11. Baker DE. Rationale for using serotonergic agents to treat irritable bowel syndrome. Am J Health Syst Pharm 2005;62:700-11.

12. Akehurst R, Kaltenthaler E. Treatment of irritable bowel syndrome: a review of randomised controlled trials. Gut 2001;48:272-82.

13. Cash BD, Chey WD. Diagnosis of irritable bowel syndrome. Gastrenterol Clin North Am 2005; 34:205-20.

14. Santos Jr JCM. Alteração congênita de fixação do segmento ceco-ascendente dá sintomas iguais aos da Síndrome do Cólon Irritável. Rev bras Coloproctol 2005; 25: 361-9.

15. Oberndorff-Klein Wooltthuis AH, Brummer RJM, De it NJ. Irritable bowel syndrome in general practice: An overview. Scand J Gastroenterol 2004: 241 suppl: 17-22.
16. Manning AP, Thompson WG, Heaton KA. Towards positive diagnosis of the irritable bowel syndrome. BMJ 1978;2: 6534.

17. Drossman DA, Corazziari E, Talley NJ, Rome II. The Functional Gastrointestinal Disorders. Diagnosis, Pathophysiology and Treatment: A Multinational Consensus. $2^{\mathrm{a}}$ ed. McLean, VA: Degnon Associates; 2000.

18. Padrón F, B. Anía. Constipation is a disease. $1^{\text {a }}$ ed. Barcelona: Lexeditorial JMS; 1997.

19. Santos Jr JCM, Cavalca AC, Caso CEQ. Mobile cecum as a syndrome: is it a myth or a fact? http://www.instmedicina.com.br/mobile.htm

( acessado em 20/abr/2005).

20. Santos Jr JCM, Cavalca AC. Caso CEQ: síndrome do ceco móvel mito ou realidade? Rev Bras Coloproctol 2004; 24:1536.

21. Guthrie E, Creed F, Dawson D, Tomenson B. A randomised controlled trial of psychotherapy in patients with refractory irritable bowel syndrome. Br J Psychiatry 1993;163: 315-21.

22. Jailwala J, Imperiale TF, Kroenke K. Pharmacologic Treatment of the Irritable Bowel Syndrome: A Systematic Review of Randomized, Controlled Trials. Ann Intern Med. 2000;133:136-47.

23. Talley NJ, Spiller R. Irritable bowel syndrome: a little understood organic bowel disease? Lancet 2002; 360:555-64.

24. Rogers RL. Mobile cecum syndrome. Dis Colon Rectum 1984;27:399-402.

25. Gwee KA. Irritable bowel syndrome in developing countriesa disorder of civilization or colonization? Neurogastroenterol Motil. 2005;17: 317-24.

26. Caldarella MP, Milano A, Laterza F. Visceral sensitivity and symptoms in patients with constipation - or diarrheapredominant irritable bowel syndrome (IBS): effect of a lowfat intraduodenal infusion. Am J Gastroenterol. 2005;100:383-9.

27. Pimentel M, Chow EJ, Lin HC. Eradication of small intestinal bacterial overgrowth reduces symptoms of irritable bowel syndrome. Am J Gastroenterol 2000;95:3503-6.

28. Pimentel M, Chow EJ, Lin HC. Normalization of lactulose breath testing correlates with symptom improvement in irritable bowel syndrome. a double-blind, randomized, placebocontrolled study. Am J Gastroenterol 2003;98: 412-9.

29. Hyams J, Colletti R, Faure C. Functional gastrointestinal disorders: Working Group Report of First World Congress of Pediatric Gastroenterology, Hepatology, Hematology, and Nutrition. J Pediatr Gastroenterol Nutr 2002;35 Suppl 2: S110-S7.

30. Tallet NJ, Fett SL, Zinmeister AR, Melton LJ III. Gastrointestinal tract symptoms and self-reported abuse: a population-based study. Gastroenterology 1994;107:1040-9.

31. Drossman DA, Leserman J, Nachman C. Sexual and physical abuse to women with functional organic gastrointestinal disorders. Ann Internal Med 1990;113: 828-30. 
32. Guthrie E, Creed E, Dawson D. A controlled trial of psychologic treatment for the irritable bowel syndrome. Gastroenterol 1991;1131:450-7.

33. Antunes RC. Abordagem da Síndrome do Cólon Irritável pela Acupuntura. VI Congresso de Acupuntura da Sociedade Medica Brasileira de Acupuntura. Gramado RS; 2002.

34. Tirol FT. Recurrent cecocolic torsion: radiological diagnosis and treatment. JSLS 2003;7:23-31.

35. Tirol FT. Cecocolic torsion. Surg Rounds 2003;26: 125-31.

36. Tirol FT. Dyspareunia: a symptom of recurrent cecocolic torsion. Abdom Surg Fall 2001: 11-5.

37. Tirol F. Recurrent cecocolic torsion: phaton tumor. Abdom Surg 1999: 20-4.

38. Tirol FT. Cecocolic torsion: classification, pathogenesis, and treatment. JSLS 2005;9:328-34.

39. Anderson JR, Welch GH. Acute volvulus of the right colon: An analysis of 69 patients. World J Surg 1986;10:336-42.

40. Gupta S, Gupta SK. Acute caecal volvulus: report of 22 cases and review of literature. Ital J Gastroenterol 1993; 25: 380-4.

41. Rothstein RD, Frank AJ, Goffner LB, Fruauff AA. Cecal volvulus: the CT whirl sign. Abdom Imaging 1993;18: 288-9.

42. Rombeau JL. Intestinal malrotation during pregnancy. Obstetrics \& Gynecology 1993;81:817-9.
43. Husain K, Fitzgerald P, Lau G. Cecal volvulus in the Cornelia de Lange syndrome. J Pediatr Surg 1994;29:1245-7.

44. Lee YJ, Lee YA, Liu TJ, Chang TH. Mobile cecum syndrome; a report of two cases. Chin Med J (Taipei) 1996;57:380-3.

45. Frischman WJ, Couper RTL, Freeman JK. Cecal Volvulus Following Gastroduodenoscopy in Cornelia de Lange Syndrome. J Pediat Gastrenterol Nutr 1996;22:205-7.

46. Yeh WC, Wang HP, Chen C. Preoperative sonographic diagnosis of midgut malrotation with volvulus in adults: the "whirlpool" sign. J Clin Ultrasound 1999; 27: 279-83.

47. Simon AM, Birnbaum BA, Jacobs JE. Isolated Infarction of the Cecum: CT Findings in Two Patients. Radiology 2000;214:513-6.

48. Moore CJ, Corl FM, Fishman EK. CT of cecal volvulus: unraveling the image. AJR Am J Roentgenol 2001; 177: 95-8.

49. Madiba TE, Thomson SR. The management of cecal volvulus. Dis Colon Rectum 2002;45:264-7.

Endereço para correspondência: JÚLIO CÉSAR M SANTOS JR.

Av. Min. Urbano Marcondes, 516 12.515-230 - Guaratinguetá (SP) 